\title{
On two congruences involving Franel numbers
}

\author{
Ji-Cai Liu \\ Department of Mathematics, Wenzhou University, Wenzhou 325035, PR China \\ jcliu2016@gmail.com
}

\begin{abstract}
Via symbolic summation method, we establish the following series for $\pi^{2}$ :

$$
\sum_{k=1}^{\infty} \frac{H_{k}-2 H_{2 k}}{(-3)^{k} k}=\frac{\pi^{2}}{18}
$$
\end{abstract}

where $H_{k}=\sum_{j=1}^{k} 1 / j$. We also derive a $p$-adic congruence related to this series. As an application, we prove two congruences involving Franel numbers, one of which was originally conjectured by Sun.

Keywords: Congruences; Harmonic numbers; Franel numbers; Bernoulli polynomials MR Subject Classifications: 11A07, 05A19, 33F10

\section{Introduction}

It is well-known that (see [16, (3)])

$$
\sum_{k=1}^{\infty} \frac{1}{k^{2}\left(\begin{array}{c}
2 k \\
k
\end{array}\right)}=\frac{\pi^{2}}{18}
$$

which can be derived from the following familiar power series expansion:

$$
2(\arcsin (x / 2))^{2}=\sum_{k=1}^{\infty} \frac{x^{2 k}}{k^{2}\left(\begin{array}{c}
2 k \\
k
\end{array}\right)} .
$$

Recall that the Euler numbers and Bernoulli polynomials are defined as

$$
\frac{2}{e^{x}+e^{-x}}=\sum_{n=0}^{\infty} E_{n} \frac{x^{n}}{n !}
$$

and

$$
\frac{x e^{t x}}{e^{x}-1}=\sum_{k=0}^{\infty} B_{k}(t) \frac{x^{k}}{k !}
$$


In 2011, Sun [19, (1.3)] showed that (1.1) possesses the following interesting $p$-adic analogue:

$$
\sum_{k=1}^{(p-1) / 2} \frac{1}{k^{2}\left(\begin{array}{c}
2 k \\
k
\end{array}\right)} \equiv(-1)^{(p-1) / 2} \frac{4}{3} E_{p-3} \quad(\bmod p),
$$

for any prime $p \geq 5$. Mattarei and Tauraso [15, Theorem 6.1] investigated congruence properties for the polynomial $\sum_{k=1}^{p-1} k^{-2}\left(\begin{array}{c}2 k \\ k\end{array}\right)^{-1} t^{k}$.

In recent decades, infinite sums on harmonic numbers related to powers of $\pi$ have been widely studied. For instance, D. Borwein and J.M. Borwein [1] proved that

$$
\sum_{k=1}^{\infty} \frac{H_{k}^{2}}{k^{2}}=\frac{17}{360} \pi^{4}
$$

where the $k$ th generalized harmonic number is given by

$$
H_{k}^{(r)}=\sum_{j=1}^{k} \frac{1}{j^{r}}
$$

with the convention that $H_{k}=H_{k}^{(1)}$. It is worth mentioning that Sun [20,25] conjectured and proved many infinite identities involving harmonic numbers related to powers of $\pi$. For more series for $\pi$ and related congruences as well as $q$-congruences, one can refer to $[2,3,6,9,13,19,21,27,28]$.

The first aim of the paper is to establish the following series for $\pi^{2}$.

Theorem 1.1 We have

$$
\sum_{k=1}^{\infty} \frac{H_{k}-2 H_{2 k}}{(-3)^{k} k}=\frac{\pi^{2}}{18}
$$

Our proof of (1.2) makes use of a finite identity, which is derived from symbolic summation method. We also show that (1.2) has the following $p$-adic analogue.

Theorem 1.2 For any prime $p \geq 5$, we have

$$
\sum_{k=1}^{(p-1) / 2} \frac{H_{k}-2 H_{2 k}}{(-3)^{k} k} \equiv \frac{1}{6}\left(\frac{p}{3}\right) B_{p-2}\left(\frac{1}{3}\right) \quad(\bmod p),
$$

where $(\dot{\overline{3}})$ denotes the Legendre symbol.

In 1894, Franel [4] found that the sums of cubes of binomial coefficients:

$$
f_{n}=\sum_{k=0}^{n}\left(\begin{array}{l}
n \\
k
\end{array}\right)^{3}
$$


satisfy the recurrence:

$$
(n+1)^{2} f_{n+1}=\left(7 n^{2}+7 n+2\right) f_{n}+8 n^{2} f_{n-1} .
$$

The numbers $f_{n}$ are known as Franel numbers, which also appear in Strehl's identity (see [18]):

$$
f_{n}=\sum_{k=0}^{n}\left(\begin{array}{l}
n \\
k
\end{array}\right)^{2}\left(\begin{array}{c}
2 k \\
n
\end{array}\right)=\sum_{k=0}^{n}\left(\begin{array}{l}
n \\
k
\end{array}\right)\left(\begin{array}{c}
k \\
n-k
\end{array}\right)\left(\begin{array}{c}
2 k \\
k
\end{array}\right) .
$$

Since the appearance of the Franel numbers, some interesting congruence properties have been gradually discovered (see [5, 11, 22, 23]). For instance, Sun [22, Theorem 1.1] proved that for any prime $p \geq 5$,

$$
\sum_{k=0}^{p-1}(-1)^{k} f_{k} \equiv\left(\frac{p}{3}\right) \quad\left(\bmod p^{2}\right)
$$

The second aim of the paper is prove the following two congruences involving Franel numbers and harmonic numbers.

Theorem 1.3 For any prime $p \geq 5$, we have

$$
\begin{aligned}
& \sum_{k=0}^{p-1}(-1)^{k} f_{k} H_{k}^{(2)} \equiv \frac{1}{2} B_{p-2}\left(\frac{1}{3}\right) \quad(\bmod p), \\
& \sum_{k=0}^{p-1}(-1)^{k} f_{k} H_{k} \equiv-2\left(\frac{p}{3}\right) q_{p}(3) \quad(\bmod p),
\end{aligned}
$$

where $q_{p}(3)$ is the Fermat quotient $\left(3^{p-1}-1\right) / p$.

Note that (1.5) was originally conjectured by Sun [24, Conjecture 57]. Our proof of (1.5) is based on Theorem 1.2. We shall prove Theorems 1.1 1.3 in Sections 2-4, respectively.

\section{Proof of Theorem 1.1}

Before proving Theorem [1.1, we require the following finite identity.

Lemma 2.1 For any non-negative integer $n$, we have

$$
\sum_{k=0}^{n}(-4)^{k}\left(\begin{array}{l}
n \\
k
\end{array}\right) \sum_{j=1}^{k} \frac{1}{j^{2}\left(\begin{array}{c}
2 j \\
j
\end{array}\right)}=(-3)^{n} \sum_{k=1}^{n} \frac{H_{k}-2 H_{2 k}}{(-3)^{k} k}
$$


Proof. By using symbolic summation package Sigma due to Schneider [17, we find that both sides of (2.1) satisfy the same recurrence:

$$
\begin{aligned}
& 3(n+1)(2 n+3) S(n)-(2 n+5)(5 n+9) S(n+1)+\left(2 n^{2}+17 n+29\right) S(n+2) \\
& +(n+3)(2 n+5) S(n+3)=0 .
\end{aligned}
$$

It is trivial to check that both sides of (2.1) are equal for $n=0,1,2$.

Proof of (1.2). By (2.1), we have

$$
\begin{aligned}
\sum_{k=1}^{n} \frac{H_{k}-2 H_{2 k}}{(-3)^{k} k} & =\left(-\frac{1}{3}\right)^{n} \sum_{j=1}^{n} \frac{1}{j^{2}\left(\begin{array}{c}
2 j \\
j
\end{array}\right)} \sum_{k=j}^{n}(-4)^{k}\left(\begin{array}{l}
n \\
k
\end{array}\right) \\
& =\left(-\frac{1}{3}\right)^{n} \sum_{j=1}^{n} \frac{1}{j^{2}\left(\begin{array}{c}
2 j \\
j
\end{array}\right)}\left((-3)^{n}-\sum_{k=0}^{j-1}(-4)^{k}\left(\begin{array}{l}
n \\
k
\end{array}\right)\right) \\
& =\sum_{j=1}^{n} \frac{1}{j^{2}\left(\begin{array}{c}
2 j \\
j
\end{array}\right)}\left(1-\sum_{k=0}^{j-1} \frac{(-4)^{k}}{(-3)^{n}}\left(\begin{array}{l}
n \\
k
\end{array}\right)\right) .
\end{aligned}
$$

It is obvious that for $0 \leq k \leq j-1$,

$$
\lim _{n \rightarrow \infty} \frac{(-4)^{k}}{(-3)^{n}}\left(\begin{array}{l}
n \\
k
\end{array}\right)=0
$$

and so

$$
\lim _{n \rightarrow \infty} \sum_{k=0}^{j-1} \frac{(-4)^{k}}{(-3)^{n}}\left(\begin{array}{l}
n \\
k
\end{array}\right)=0
$$

Letting $n \rightarrow \infty$ on both sides of (2.2) and noting (2.3), we arrive at

$$
\sum_{k=1}^{\infty} \frac{H_{k}-2 H_{2 k}}{(-3)^{k} k}=\sum_{j=1}^{\infty} \frac{1}{j^{2}\left(\begin{array}{c}
2 j \\
j
\end{array}\right)} \text {. }
$$

Then the proof of (1.2) follows from (1.1) and (2.4).

\section{Proof of Theorem 1.2}

In order to prove Theorem 1.2, we need three preliminary results.

Lemma 3.1 For any non-negative integer n, we have

$$
\left(\begin{array}{c}
2 n \\
n
\end{array}\right) \sum_{k=1}^{n} \frac{1}{k^{2}\left(\begin{array}{c}
2 k \\
k
\end{array}\right)}=\left(\begin{array}{c}
2 n \\
n
\end{array}\right) H_{n}^{(2)}+\sum_{k=1}^{n} \frac{-3\left(\frac{k}{3}\right)^{2}+2}{k^{2}}\left(\begin{array}{c}
2 n \\
n+k
\end{array}\right) .
$$


Proof. An identity due to Mattarei and Tauraso [15, (22)] says

$$
\left(\begin{array}{c}
2 n \\
n
\end{array}\right) \sum_{k=1}^{n} \frac{t^{k}}{k^{2}\left(\begin{array}{c}
2 k \\
k
\end{array}\right)}=\left(\begin{array}{c}
2 n \\
n
\end{array}\right) H_{n}^{(2)}+\sum_{k=1}^{n} \frac{v_{k}(t-2)}{k^{2}}\left(\begin{array}{c}
2 n \\
n+k
\end{array}\right),
$$

where the Lucas sequences $\left\{v_{k}(t)\right\}_{k \geq 0}$ are defined by

$$
v_{0}(t)=2, \quad v_{1}(t)=t, \quad \text { and } \quad v_{k}(t)=t v_{k-1}(t)-v_{k-2}(t) \quad \text { for } k \geq 2 \text {. }
$$

It is not hard to check that

$$
v_{k}(-1)=-3\left(\frac{k}{3}\right)^{2}+2
$$

Letting $t=1$ in (3.2) and noting (3.3), we reach (3.1).

Lemma 3.2 For any prime $p \geq 5$, we have

$$
\sum_{k=0}^{(p-1) / 2}\left(\begin{array}{c}
2 k \\
k
\end{array}\right) H_{k}^{(2)} \equiv-\frac{1}{6} B_{p-2}\left(\frac{1}{3}\right) \quad(\bmod p) .
$$

Proof. We begin with the following polynomial congruence [15, (37)]:

$$
\sum_{k=1}^{p-1} t^{p-k}\left(\begin{array}{c}
2 k \\
k
\end{array}\right) H_{k}^{(2)} \equiv-2 t \sum_{k=1}^{p-1} \frac{u_{k}(2-t)}{k^{2}} \quad(\bmod p),
$$

where the Lucas sequences $\left\{u_{k}(t)\right\}_{k \geq 0}$ are defined by

$$
u_{0}(t)=0, \quad u_{1}(t)=1, \quad \text { and } \quad u_{k}(t)=t u_{k-1}(t)-u_{k-2}(t) \quad \text { for } k \geq 2 .
$$

It is trivial to check that

$$
u_{k}(1)=\frac{(-1)^{\left\lfloor\frac{k}{3}\right\rfloor}+(-1)^{\left\lfloor\frac{k-1}{3}\right\rfloor}}{2},
$$

where $\lfloor x\rfloor$ denotes the integral part of real $x$. Letting $t=1$ in (3.5) and using (3.6) gives

$$
\sum_{k=1}^{p-1}\left(\begin{array}{c}
2 k \\
k
\end{array}\right) H_{k}^{(2)} \equiv-\sum_{k=1}^{p-1} \frac{(-1)^{\left\lfloor\frac{k}{3}\right\rfloor}+(-1)^{\left\lfloor\frac{k-1}{3}\right\rfloor}}{k^{2}}(\bmod p) .
$$


Note that

$$
\begin{aligned}
& \sum_{k=1}^{p-1} \frac{(-1)^{\left\lfloor\frac{k}{3}\right\rfloor}+(-1)^{\left\lfloor\frac{k-1}{3}\right\rfloor}}{k^{2}} \\
& =2\left(\sum_{\substack{1 \leq k \leq p-1 \\
k \equiv 1}} \frac{1}{k^{2}}+\sum_{\substack{1 \leq k \leq p-1 \\
k \equiv 2}} \frac{1}{k^{2}}-\sum_{\substack{1 \leq k \leq p-1 \\
k \equiv 4}} \frac{1}{k^{2}}-\sum_{\substack{1 \leq k \leq p-1 \\
k \equiv 5}} \frac{1}{\bmod ^{2}}\right) \\
& =2 H_{p-1}^{(2)}-2 \sum_{\substack{1 \leq k \leq p-1 \\
(\bmod 3)}} \frac{1}{k^{2}-4}\left(\sum_{\substack{1 \leq k \leq p-1 \\
k \equiv 4}} \frac{1}{k^{2}}+\sum_{\substack{1 \leq k \leq p-1 \\
(\bmod 6)}} \frac{1}{k^{2}}\right) \\
& =2 H_{p-1}^{(2)}-\frac{2}{9} H_{\lfloor p / 3\rfloor}^{(2)}-\frac{1}{9}\left(\sum_{k=1}^{\left\lfloor\frac{\bmod 6)}{6}\right\rfloor} \frac{1}{(k-1 / 3)^{2}}+\sum_{k=1}^{\left\lfloor\frac{p}{6}\right\rfloor} \frac{1}{(k-1 / 6)^{2}}\right) .
\end{aligned}
$$

If $p \equiv 1(\bmod 6)$, then $\lfloor p / 6\rfloor \equiv-1 / 6(\bmod p)$, and so

$$
\begin{aligned}
& \sum_{k=1}^{\left\lfloor\frac{p+1}{6}\right\rfloor} \frac{1}{(k-1 / 3)^{2}}+\sum_{k=1}^{\left\lfloor\frac{p}{6}\right\rfloor} \frac{1}{(k-1 / 6)^{2}} \\
& \equiv \sum_{k=1}^{\left\lfloor\frac{p}{6}\right\rfloor} \frac{1}{(2\lfloor p / 6\rfloor+k)^{2}}+\sum_{k=1}^{\left\lfloor\frac{p}{6}\right\rfloor} \frac{1}{(\lfloor p / 6\rfloor+k)^{2}} \quad(\bmod p) \\
& =H_{(p-1) / 2}^{(2)}-H_{\lfloor p / 6\rfloor}^{(2)} .
\end{aligned}
$$

It follows from (3.8) and (3.9) that

$$
\sum_{k=1}^{p-1} \frac{(-1)^{\left\lfloor\frac{k}{3}\right\rfloor}+(-1)^{\left\lfloor\frac{k-1}{3}\right\rfloor}}{k^{2}} \equiv 2 H_{p-1}^{(2)}-\frac{2}{9} H_{\lfloor p / 3\rfloor}^{(2)}-\frac{1}{9} H_{(p-1) / 2}^{(2)}+\frac{1}{9} H_{\lfloor p / 6\rfloor}^{(2)} \quad(\bmod p) .
$$

By Wolstenholme's theorem [10, page 114], [19, Lemma 2.4], [12, (9)] and the congruence 
below equation [12, (47)], we have

$$
\begin{aligned}
& H_{p-1}^{(2)} \equiv 0 \quad(\bmod p), \\
& H_{(p-1) / 2}^{(2)} \equiv 0 \quad(\bmod p), \\
& H_{\lfloor p / 3\rfloor}^{(2)} \equiv \frac{1}{2}\left(\frac{p}{3}\right) B_{p-2}\left(\frac{1}{3}\right) \quad(\bmod p), \\
& H_{\lfloor p / 6\rfloor}^{(2)} \equiv 5 H_{\lfloor p / 3\rfloor}^{(2)} \stackrel{(3.13)}{\equiv} \frac{5}{2}\left(\frac{p}{3}\right) B_{p-2}\left(\frac{1}{3}\right) \quad(\bmod p) .
\end{aligned}
$$

Substituting (3.11)-(3.14) into (3.10) gives

$$
\sum_{k=1}^{p-1} \frac{(-1)^{\left\lfloor\frac{k}{3}\right\rfloor}+(-1)^{\left\lfloor\frac{k-1}{3}\right\rfloor}}{k^{2}} \equiv \frac{1}{6} B_{p-2}\left(\frac{1}{3}\right) \quad(\bmod p) .
$$

If $p \equiv 5(\bmod 6)$, in a similar way, we can also prove that

$$
\sum_{k=1}^{p-1} \frac{(-1)^{\left\lfloor\frac{k}{3}\right\rfloor}+(-1)^{\left\lfloor\frac{k-1}{3}\right\rfloor}}{k^{2}} \equiv \frac{1}{6} B_{p-2}\left(\frac{1}{3}\right) \quad(\bmod p) .
$$

Finally, combining (3.7), (3.15) and (3.16), we arrive at

$$
\sum_{k=1}^{p-1}\left(\begin{array}{c}
2 k \\
k
\end{array}\right) H_{k}^{(2)} \equiv-\frac{1}{6} B_{p-2}\left(\frac{1}{3}\right) \quad(\bmod p),
$$

as desired.

Lemma 3.3 Let $p \geq 5$ be a prime. Then for $0 \leq d \leq \frac{p-1}{2}$, we have

$$
\sum_{k=0}^{(p-1) / 2}\left(\begin{array}{c}
2 k \\
k+d
\end{array}\right) \equiv\left(\frac{p-d}{3}\right)-\left(\frac{d}{3}\right) \quad(\bmod p)
$$

Proof. From [26, Theorem 4.2], we deduce that for $0 \leq d \leq n$,

$$
\sum_{k=0}^{n-1}\left(\begin{array}{c}
2 k \\
k+d
\end{array}\right)=\sum_{k=0}^{n-d}\left(\frac{n-d-k}{3}\right)\left(\begin{array}{c}
2 n \\
k
\end{array}\right) .
$$

Letting $n=(p+1) / 2$ in the above gives

$$
\sum_{k=0}^{(p-1) / 2}\left(\begin{array}{c}
2 k \\
k+d
\end{array}\right)=\sum_{k=0}^{(p+1) / 2-d}\left(\frac{(p+1) / 2-d-k}{3}\right)\left(\begin{array}{c}
p+1 \\
k
\end{array}\right) .
$$


Since for $2 \leq k \leq(p+1) / 2-d$, we have

$$
\left(\begin{array}{c}
p+1 \\
k
\end{array}\right) \equiv 0 \quad(\bmod p)
$$

and so

$$
\sum_{k=0}^{(p-1) / 2}\left(\begin{array}{c}
2 k \\
k+d
\end{array}\right) \equiv\left(\frac{(p+1) / 2-d}{3}\right)+\left(\frac{(p+1) / 2-d-1}{3}\right) \quad(\bmod p) .
$$

Next, we distinguish two cases $p \equiv 1(\bmod 6)$ and $p \equiv 5(\bmod 6)$ to prove (3.17)). In both cases, we can show that

$$
\left(\frac{(p+1) / 2-d}{3}\right)+\left(\frac{(p+1) / 2-d-1}{3}\right) \equiv\left(\frac{p-d}{3}\right)-\left(\frac{d}{3}\right) \quad(\bmod p) .
$$

Finally, combining (3.18) and (3.19), we complete the proof of (3.17).

Now we are ready to prove Theorem 1.2 . Proof of (1.3) . Letting $n=\frac{p-1}{2}$ in (2.1) and noting that

$$
\left(\begin{array}{c}
(p-1) / 2 \\
k
\end{array}\right) \equiv \frac{\left(\begin{array}{c}
2 k \\
k
\end{array}\right)}{(-4)^{k}} \quad(\bmod p) \quad \text { and } \quad(-3)^{(p-1) / 2} \equiv\left(\frac{p}{3}\right) \quad(\bmod p)
$$

we obtain

$$
\sum_{k=0}^{(p-1) / 2}\left(\begin{array}{c}
2 k \\
k
\end{array}\right) \sum_{j=1}^{k} \frac{1}{j^{2}\left(\begin{array}{c}
2 j \\
j
\end{array}\right)} \equiv\left(\frac{p}{3}\right) \sum_{k=1}^{(p-1) / 2} \frac{H_{k}-2 H_{2 k}}{(-3)^{k} k} \quad(\bmod p) .
$$

In order to prove (1.3), it suffices to show that

$$
\sum_{k=0}^{(p-1) / 2}\left(\begin{array}{c}
2 k \\
k
\end{array}\right) \sum_{j=1}^{k} \frac{1}{j^{2}\left(\begin{array}{c}
2 j \\
j
\end{array}\right)} \equiv \frac{1}{6} B_{p-2}\left(\frac{1}{3}\right) \quad(\bmod p) .
$$

By (3.1), (3.4) and (3.17), we have

$$
\begin{aligned}
& \sum_{k=0}^{(p-1) / 2}\left(\begin{array}{c}
2 k \\
k
\end{array}\right) \sum_{j=1}^{k} \frac{1}{j^{2}\left(\begin{array}{c}
2 j \\
j
\end{array}\right)} \\
& \equiv-\frac{1}{6} B_{p-2}\left(\frac{1}{3}\right)+\sum_{k=0}^{(p-1) / 2} \sum_{j=1}^{k} \frac{-3\left(\frac{j}{3}\right)^{2}+2}{j^{2}}\left(\begin{array}{c}
2 k \\
k+j
\end{array}\right) \quad(\bmod p) \\
& =-\frac{1}{6} B_{p-2}\left(\frac{1}{3}\right)+\sum_{j=1}^{(p-1) / 2} \frac{-3\left(\frac{j}{3}\right)^{2}+2}{j^{2}} \sum_{k=0}^{(p-1) / 2}\left(\begin{array}{c}
2 k \\
k+j
\end{array}\right) \\
& \equiv-\frac{1}{6} B_{p-2}\left(\frac{1}{3}\right)+\sum_{j=1}^{(p-1) / 2} \frac{\left(-3\left(\frac{j}{3}\right)^{2}+2\right)\left(\left(\frac{p-j}{3}\right)-\left(\frac{j}{3}\right)\right)}{j^{2}}(\bmod p) .
\end{aligned}
$$


If $p \equiv 1(\bmod 6)$, then $\lfloor p / 6\rfloor \equiv-1 / 6(\bmod p)$, and so

$$
\begin{aligned}
& \sum_{j=1}^{(p-1) / 2} \frac{\left(-3\left(\frac{j}{3}\right)^{2}+2\right)\left(\left(\frac{p-j}{3}\right)-\left(\frac{j}{3}\right)\right)}{j^{2}} \\
& =\sum_{\substack{1 \leq j \leq(p-1) / 2 \\
j \equiv 1 \quad(\bmod 3)}} \frac{1}{j^{2}}+2 \sum_{\substack{1 \leq j \leq(p-1) / 2 \\
j \equiv 0 \quad(\bmod 3)}} \frac{1}{j^{2}} \\
& =H_{(p-1) / 2}^{(2)}+\frac{1}{9} H_{\lfloor p / 6\rfloor}^{(2)}-\sum_{\substack{1 \leq j \leq(p-1) / 2 \\
j \equiv 2}} \frac{1}{j^{2}} .
\end{aligned}
$$

Note that

$$
\begin{aligned}
\sum_{\substack{1 \leq j \leq(p-1) / 2 \\
j \equiv 2(\bmod 3)}} \frac{1}{j^{2}} & =\frac{1}{9} \sum_{j=1}^{\left\lfloor\frac{p}{6}\right\rfloor} \frac{1}{(j-1 / 3)^{2}} \\
& \equiv \frac{1}{9} \sum_{j=1}^{\left\lfloor\frac{p}{6}\right\rfloor} \frac{1}{(j+2\lfloor p / 6\rfloor)^{2}} \quad(\bmod p) \\
& =\frac{1}{9}\left(H_{(p-1) / 2}^{(2)}-H_{\lfloor p / 3\rfloor}^{(2)}\right) .
\end{aligned}
$$

It follows from (3.12), (3.13), (3.14), (3.22) and (3.23) that

$$
\begin{aligned}
\sum_{j=1}^{(p-1) / 2} \frac{\left(-3\left(\frac{j}{3}\right)^{2}+2\right)\left(\left(\frac{p-j}{3}\right)-\left(\frac{j}{3}\right)\right)}{j^{2}} & \equiv \frac{8}{9} H_{(p-1) / 2}^{(2)}+\frac{1}{9} H_{\lfloor p / 3\rfloor}^{(2)}+\frac{1}{9} H_{\lfloor p / 6\rfloor}^{(2)} \\
& \equiv \frac{1}{3} B_{p-2}\left(\frac{1}{3}\right) \quad(\bmod p) .
\end{aligned}
$$

If $p \equiv 5(\bmod 6)$, in a similar way, we can also prove that

$$
\sum_{j=1}^{(p-1) / 2} \frac{\left(-3\left(\frac{j}{3}\right)^{2}+2\right)\left(\left(\frac{p-j}{3}\right)-\left(\frac{j}{3}\right)\right)}{j^{2}} \equiv \frac{1}{3} B_{p-2}\left(\frac{1}{3}\right) \quad(\bmod p) .
$$

Finally, combining (3.21), (3.24) and (3.25), we reach (3.20). 


\section{Proof of Theorem 1.3}

To prove Theorem 1.3, we require the following two identities.

Lemma 4.1 For any non-negative integer $n$, we have

$$
\begin{aligned}
& \sum_{k=n}^{2 n}(-1)^{k}\left(\begin{array}{l}
k \\
n
\end{array}\right)\left(\begin{array}{c}
n \\
k-n
\end{array}\right) H_{k}^{(2)}=3 \sum_{k=1}^{n} \frac{1}{k^{2}\left(\begin{array}{c}
2 k \\
k
\end{array}\right)} \\
& \sum_{k=n}^{2 n}(-1)^{k}\left(\begin{array}{l}
k \\
n
\end{array}\right)\left(\begin{array}{c}
n \\
k-n
\end{array}\right) H_{k}=2 H_{n} .
\end{aligned}
$$

Proof. The identities (4.1) and (4.2) possess the same proofs as (2.1), and we omit the details.

Proof of (1.5). By (1.4), we have

$$
\begin{aligned}
\sum_{k=0}^{p-1}(-1)^{k} f_{k} H_{k}^{(2)} & =\sum_{k=0}^{p-1}(-1)^{k} H_{k}^{(2)} \sum_{j=0}^{k}\left(\begin{array}{c}
k \\
j
\end{array}\right)\left(\begin{array}{c}
j \\
k-j
\end{array}\right)\left(\begin{array}{c}
2 j \\
j
\end{array}\right) \\
& =\sum_{j=0}^{p-1}\left(\begin{array}{c}
2 j \\
j
\end{array}\right) \sum_{k=0}^{p-1}(-1)^{k}\left(\begin{array}{c}
k \\
j
\end{array}\right)\left(\begin{array}{c}
j \\
k-j
\end{array}\right) H_{k}^{(2)} \\
& \equiv \sum_{j=0}^{(p-1) / 2}\left(\begin{array}{c}
2 j \\
j
\end{array}\right) \sum_{k=0}^{p-1}(-1)^{k}\left(\begin{array}{c}
k \\
j
\end{array}\right)\left(\begin{array}{c}
j \\
k-j
\end{array}\right) H_{k}^{(2)} \quad(\bmod p),
\end{aligned}
$$

because $\left(\begin{array}{c}2 j \\ j\end{array}\right) \equiv 0(\bmod p)$ for $(p+1) / 2 \leq j \leq p-1$.

Note that for $0 \leq j \leq(p-1) / 2$,

$$
\begin{aligned}
\sum_{k=0}^{p-1}(-1)^{k}\left(\begin{array}{c}
k \\
j
\end{array}\right)\left(\begin{array}{c}
j \\
k-j
\end{array}\right) H_{k}^{(2)} & =\sum_{k=0}^{2 j}(-1)^{k}\left(\begin{array}{c}
k \\
j
\end{array}\right)\left(\begin{array}{c}
j \\
k-j
\end{array}\right) H_{k}^{(2)} \\
& \stackrel{\text { 4.11) }}{=} 3 \sum_{k=1}^{j} \frac{1}{k^{2}\left(\begin{array}{c}
2 k \\
k
\end{array}\right)} .
\end{aligned}
$$

It follows from (4.3), (4.4) and (3.20) that

$$
\begin{aligned}
\sum_{k=0}^{p-1}(-1)^{k} f_{k} H_{k}^{(2)} & \equiv 3 \sum_{j=0}^{(p-1) / 2}\left(\begin{array}{c}
2 j \\
j
\end{array}\right) \sum_{k=1}^{j} \frac{1}{k^{2}\left(\begin{array}{c}
2 k \\
k
\end{array}\right)} \\
& \equiv \frac{1}{2} B_{p-2}\left(\frac{1}{3}\right) \quad(\bmod p),
\end{aligned}
$$

as desired. 
Proof of (1.6). Similarly to the proof of (1.5), by using (4.2), we can show that

$$
\sum_{k=0}^{p-1}(-1)^{k} f_{k} H_{k} \equiv 2 \sum_{j=0}^{(p-1) / 2}\left(\begin{array}{c}
2 j \\
j
\end{array}\right) H_{j} \quad(\bmod p) .
$$

Combining the above and the following congruence (see [14, page 528]):

$$
\sum_{j=0}^{(p-1) / 2}\left(\begin{array}{c}
2 j \\
j
\end{array}\right) H_{j} \equiv-\left(\frac{p}{3}\right) q_{p}(3) \quad(\bmod p),
$$

we complete the proof of $(1.6)$.

Acknowledgments. This work was supported by the National Natural Science Foundation of China (grant 11801417).

\section{References}

[1] D. Borwein and J.M. Borwein, On an intriguing integral and some series related to $\zeta(4)$, Proc. Amer. Math. Soc. 123 (1995), 1191-1198.

[2] H.H. Chan, S.H. Chan and Z. Liu, Domb's numbers and Ramanujan-Sato type series for 1/ $\pi$, Adv. Math. 186 (2004), 396-410.

[3] H.H. Chan, J. Wan and W. Zudilin, Legendre polynomials and Ramanujan-type series for 1/ $\pi$, Israel J. Math. 194 (2013), 183-207.

[4] J. Franel, On a question of Laisant, L'Intermédiaire des Mathématiciens 1 (1894), 45-47.

[5] V.J.W. Guo, Proof of two conjectures of Sun on congruences for Franel numbers, Integral Transforms Spec. Funct. 24 (2013), 532-539.

[6] V.J.W. Guo, Proof of a generalization of the (B.2) supercongruence of Van Hamme through a $q$-microscope, Adv. in Appl. Math., to appear.

[7] V.J.W. Guo and M. J. Schlosser, Some new q-congruences for truncated basic hypergeometric series: even powers, Results Math. 75 (2020), Art. 1.

[8] V.J.W. Guo and M.J. Schlosser, Some $q$-supercongruences from transformation formulas for basic hypergeometric series, Constr. Approx., to appear.

[9] V.J.W. Guo and W. Zudilin, A q-microscope for supercongruences, Adv. Math. 346 (2019), 329-358.

[10] G.H. Hardy and E.M. Wright, An Introduction to the Theory of Numbers, sixth ed., Oxford University Press, Oxford, 2008.

[11] F. Jarvis and H.A. Verrill, Supercongruences for the Catalan-Larcombe-French numbers, Ramanujan J. 22 (2010), 171-186.

[12] E. Lehmer, On congruences involving Bernoulli numbers and the quotients of Fermat and Wilson, Ann. Math. 39 (1938), 350-360.

[13] J.-C. Liu and F. Petrov, Congruences on sums of $q$-binomial coefficients, Adv. in Appl. Math. 116 (2020), 102003. 
[14] G.-S. Mao and Z.-W. Sun, Two congruences involving harmonic numbers with applications, Int. J. Number Theory 12 (2016), 527-539.

[15] S. Mattarei and R. Tauraso, Congruences for central binomial sums and finite polylogarithms, J. Number Theory 133 (2013), 131-157.

[16] A. van der Poorten, A proof that Euler missed...Apéry's proof of the irrationality of $\zeta(3)$, Math. Intelligencer 1 (1978/79), 195-203.

[17] C. Schneider, Symbolic summation assists combinatorics, Sém. Lothar. Combin. 56 (2007), B56b, 36 pp.

[18] V. Strehl, Binomial sums and identities, Maple Technical Newsletter 10 (1993), 37-49.

[19] Z.-W. Sun, Super congruences and Euler numbers, Sci. China Math. 54 (2011), 2509-2535.

[20] Z.-W. Sun, List of conjectural series for powers of $\pi$ and other constants, preprint (2011), arXiv:1102.5649.

[21] Z.-W. Sun, Arithmetic theory of harmonic numbers, Proc. Amer. Math. Soc. 140 (2012), 415-428.

[22] Z.-W. Sun, Congruences for Franel numbers, Adv. in Appl. Math. 51 (2013), 524-535.

[23] Z.-W. Sun, Connections between $p=x^{2}+3 y^{2}$ and Franel numbers, J. Number Theory 133 (2013), 2914-2928.

[24] Z.-W. Sun, Open conjectures on congruences, J. Nanjing Univ. Math. Biquart. 36 (2019), $1-99$.

[25] Z.-W. Sun, New series for powers of $\pi$ and related congruences, preprint (2019), arXiv:1911.05456.

[26] R. Tauraso, q-Analogs of some congruences involving Catalan numbers, Adv. in Appl. Math. 48 (2012), 603-614.

[27] X. Wang and X. Yuan, Infinite summation formulas involving Riemann-Zeta function, preprint (2019), arXiv:1908.09468,

[28] W. Zudilin, Ramanujan-type supercongruences, J. Number Theory 129 (2009), 1848-1857. 